\title{
Nuevos anticoagulantes: ¿cuántos de nuestros pacientes anticoagulados pueden tomarlos?
}

\author{
Pedro Domínguez Sánchez-Migallón
}

Médico de Familia. Centro de Salud Manzanares II.

\section{Correspondencia: Pedro Domínguez Sánchez-Migallón. Centro de Salud Manzanares II. Carretera de la Solana $s / n$. Manzanares (Ciudad Real). Correo electrónico: pdsanchez@sescam.jccm.es}

Recibido el 23 de julio de 2012.

Aceptado para su publicación el 14 de agosto de 2012.

\section{RESUMEN}

Objetivo: Conocer el porcentaje de pacientes con fibrilación auricular no valvular (FANV) y Terapia Anticoagulante Oral susceptibles de cambiar a los nuevos anticoagulantes orales, y en cuantos de ellos el cambio sería coste-efectivo.

Material y Métodos: Estudio observacional, descriptivo y transversal en el ámbito de la Atención Primaria (Centro de Salud de Manzanares II) sobre 94 pacientes (52 varones y 42 mujeres) con factor de riesgo "anticoagulación" y FANV, mediante recogida de datos de historia clínica (sociodemográficos, clínicos, analíticos) y aplicación de las escalas CHADS2 y CHAD2DS2VASc, valorando la indicación según las puntuaciones.

Resultados: El 92,5\% son mayores de 65 años y el $66 \%$ son mayores de 75 años. Hay una elevada prevalencia de Hipertensión Arterial $(78,7 \%)$, Diabetes Mellitus $(41,4 \%)$, Enfermedad Renal Crónica (ERC) (34\%), Insuficiencia Cardiaca (26,5\%) y Trombopenia (11,7\%). El 4,2\% tienen un filtrado glomerular menor de $30 \mathrm{ml} / \mathrm{min}$, y el $12,7 \%$ entre $30-50 \mathrm{ml} / \mathrm{min}$. De los mayores de 65 años, tiene HTA el 67\%, Diabetes Mellitus el 35,1\% y Enfermedad Coronaria el 21,2\%. Valores de la escala CHADS2 (2,46 $\pm 1,27$ puntos) con $>2$ puntos $(76,5 \%)$. Toman fármacos con posible interacción (amiodarona o verapamilo) en el $5,3 \%$. El INR está en $<60 \%$ de Tiempo en Rango Terapéutico en el 40,2\% (IC95\%: 30,4-51,0) de los pacientes, por lo que unido a los valores de CHADS2 (1, 2 ó $\geq 3$ puntos: 5,3\%, 10,6 y 17\% respectivamente) hace que el nuevo anticoagulante pueda ser coste-efectivo hasta en el $32,9 \%$ de los pacientes.

Conclusiones: Los nuevos anticoagulantes orales podrían estar indicados hasta en dos de cada tres pacientes con FANV. Estarían contraindicados en un $4 \%$ de los casos y habría que tener precaución con su uso en uno de cada ocho pacientes, por alteración en la función renal. Su empleo sería coste-efectivo hasta en uno de cada tres pacientes.

Palabras clave: Anticoagulación, Acenocumarol, Fibrilación Auricular No Valvular.

\section{ABSTRACT}

New anticoagulants: how many of our anticoagulated patients can take them?

Objective. To establish the percentage of patients with non-valvular atrial fibrillation (AF) on Oral Anticoagulant Therapy subject to new oral anticoagulants, and in how many of them the change would be cost-effective.

Material and Methods. Observational, descriptive and cross-sectional study in the area of primary care (Centro de Salud de Manzanares II) on 94 patients (52 males and 42 female) with "anticoagulation" and atrial fibrillation (AF) risk factor by the collection of clinical history (sociodemographic, clinical analytical) data and application of CHADS2 y CHAD2DS2-VASc scores, assessing prescription based on scores.

Results. $92,5 \%$ are over 65 years of age and $66 \%$ over 75 . There is a very high rate of High Blood Pressure (78,7\%), Diabetes Mellitus (41,4\%), Chronic Kidney Disease (34\%), Heart Failure $(26,5 \%)$ and Thrombopenia (11,7\%). 4,2\% have a glomerular filtration below $30 \mathrm{ml} / \mathrm{min}$ and $12,7 \%$ between $30-50 \mathrm{ml} / \mathrm{min}$. Out of those over $65,67 \%$ have High Blood Pressure, $35,1 \%$ Diabetes Mellitus and $21,2 \%$, Heart Disease. CHADS2 scale values $(2,46 \pm 1.27$ points) with $\geq 2$ points $(76,5 \%)$. They take drugs with a possible interaction (amiodarone or verapamil) of $5,3 \%$. The INR is at $<60 \%$ of Therapeutic Range Time at $40,2 \%$ (IC95\%: 30,4-51,0) of patients which, coupled with the CHADS2 values (1,2 or $\geq 3$ points: $5,3 \%, 10.6$ y $17 \%$ respectively), makes the new anticoagulant cost-effective in $32,9 \%$ of patients.

Conclusions. The new oral anticoagulants could be indicated in up to two out of three patients with non-valvular AF. They would be contraindicated in $4 \%$ of cases, and precaution is necessary in one out of eight patients, due to impaired renal function. Its use would be cost-effective in up to one out of three patients.

Key words: Anticoagulation, Acenocumarol, NonValvular Atrial Fibrillation 


\section{INTRODUCCIÓN}

La anticoagulación, generalmente realizada en España con Acenocumarol (Sintrom ${ }^{\circledR} 1$ y $4 \mathrm{mg}$ ), es una herramienta terapéutica indicada en la prevención de complicaciones tromboembólicas. El número de pacientes con tratamiento anticoagulante oral (TAO) ha crecido notablemente en los últimos años de modo que, en el estudio de Navarro ${ }^{1}$, están en tratamiento con TAO 13,19 pacientes/1000 habitantes, siendo la principal indicación la Fibrilación Auricular $(47,1 \%$ de los pacientes). Además, el TAO con acenocumarol no está exento de riesgos, interacciones con alimentos y medicamentos, así como complicaciones (a veces mortales), siendo las hemorragias cerebrales las que ocasionan mayor número de fallecimientos, mientras que las hemorragias digestivas son las que generan mayor número de ingresos hospitalarios, precisando ajuste de dosis mediante la determinación del INR (en sangre venosa o capilar), considerándose internacionalmente como aceptable cuando el $70 \%$ de los controles se encuentran dentro del rango terapéutico².

La Fibrilación Auricular (FA), cuya prevalencia en consultas de Atención Primaria (AP) es del $6,1 \%^{3}$, no es algo estático, sino que evoluciona en el tiempo, de tal modo que más del $75 \%$ de la FA paroxística sufrirán FA permanente tras 14 años de seguimiento ${ }^{4}$, ya que la FA produce un remodelado auricular, inicialmente eléctrico y estructural después, que predispone a que se perpetúe. El riesgo tromboembólico es el mismo, independientemente del tipo de FA (paroxística, persistente o permanente) o de si es sintomática o no, por lo que hay estratificar adecuadamente el dicho riesgo e instaurar el tratamiento antitrombótico apropiado 5 . A pesar de ello, el tratamiento anticoagulante en la FA atendida en AP dista mucho de ser óptimo ${ }^{3}$, de modo que una revisión sistemática sobre este tema ha subrayado la infrautilización del TAO en pacientes con FA y un riesgo elevado de ictus en la práctica clínica real ${ }^{6}$.

Existen escalas que miden el riesgo de complicaciones tromboembólicas en paciente con FA no valvular. La más utilizada es la escala CHADS2, con un mínimo de 0 puntos y un máximo de 6 puntos, que establece la indicación de uso de antiagregante 0 anticoagulante (tablas 1 y 2) ${ }^{7}$. La nueva escala CHA2DS2-VASc ${ }^{5}$, que otorga un punto a la presencia de insuficiencia cardiaca o disfunción, hipertensión arterial, diabetes, enfermedad vascular y sexo femenino, y dos puntos a edad $\geq 75$ años e ictus, tiene mejor valor

\begin{tabular}{|c|c|c|c|c|c|}
\hline Letra & Escala CHADS $_{2}$ & Puntos & Letra & Escala HASBLED & Puntos \\
\hline C & Insuficiencia cardíaca & 1 & $\mathbf{H}$ & Hipertensión arterial & $\overline{1}$ \\
\hline H & Hipertensión arterial & 1 & A & $\begin{array}{l}\text { Función renal o hepática alteradas (1 punto } \\
\text { por cada) }\end{array}$ & 1 ó 2 \\
\hline A & Edad $\geq 75$ años & 1 & $\mathbf{S}$ & Accidente cerebrovascular & 1 \\
\hline D & Diabetes Mellitus & 1 & B & Sangrado & 1 \\
\hline $\mathbf{s}$ & Ictus previo & 2 & $\mathbf{L}$ & INR lábil & 1 \\
\hline \multirow{3}{*}{\multicolumn{3}{|c|}{ Máximo 6 puntos }} & $\mathbf{E}$ & Edad avanzada (>65 años) & 1 \\
\hline & & & D & $\begin{array}{l}\text { Fármacos o alcohol } \\
\text { (1 punto por cada) }\end{array}$ & 1 ó 2 \\
\hline & & & \multicolumn{3}{|c|}{ Máximo 9 puntos } \\
\hline
\end{tabular}

Tabla 1. Riesgo tromboembólico (Escala CHADS2) y de sangrado (Escala HASBLED)

\begin{tabular}{c|c|l}
\hline Escala CHADS2 & Nivel de Riesgo & Recomendación de tratamiento \\
\hline 0 & Bajo & Acido acetilsalicílico (AAS) (75-325 mg/día) \\
1 & Bajo a Moderado & $\begin{array}{l}\text { Anticoagulación (INR de 2 a 3) ó AAS (75-325 mg/día) } \\
\text { Anticoagulación (INR de 2 a 3) }\end{array}$ \\
\hline
\end{tabular}

Tabla 2. Nivel de riesgo tromboembólico (Escala CHADS2) y terapia anticoagulante. 


\begin{tabular}{|c|c|c|c|c|}
\hline Característica & $\begin{array}{l}\text { Total } \\
(\mathrm{n}=94)\end{array}$ & $\begin{array}{c}\text { Varones } \\
(n=52)\end{array}$ & $\begin{array}{c}\text { Mujeres } \\
(n=42)\end{array}$ & Significación \\
\hline $\begin{array}{l}\text { Edad (años) }(x \pm D E) \\
\text { Edad } \geq 65 \text { años }(\%)\end{array}$ & $\begin{array}{c}77(8,4) \\
87(92,5 \%)\end{array}$ & $\begin{array}{c}74,7(9,3) \\
45(86,5 \%)\end{array}$ & $\begin{array}{c}79,8(6,1) \\
42(100 \%)\end{array}$ & $\begin{array}{l}<0,01 \\
\text { NS }\end{array}$ \\
\hline $\begin{array}{ll}\text { Factores Riesgo: } & \text { HTA } \\
& \text { DM2 } \\
& \text { Dislipemia } \\
& \text { Tabaquismo } \\
& \text { Obesidad }\end{array}$ & $\begin{array}{c}78,7 \% \\
41,2 \% \\
40,4 \% \\
4,2 \% \\
58,5 \%\end{array}$ & $\begin{array}{c}71,1 \% \\
38,4 \% \\
32,6 \% \\
5,7 \% \\
53,8 \%\end{array}$ & $\begin{array}{c}88,0 \% \\
45,2 \% \\
50 \% \\
2,3 \% \\
64,3 \%\end{array}$ & $\begin{array}{l}<0,05 \\
\text { NS } \\
\text { NS } \\
\text { NS } \\
\text { NS }\end{array}$ \\
\hline $\begin{array}{c}\text { Comorbilidades: EPOC } \\
\text { C. Isquémica } \\
\text { I. Cardíaca }\end{array}$ & $\begin{array}{l}12,7 \% \\
23,4 \% \\
26,5 \%\end{array}$ & $\begin{array}{c}23 \% \\
19,2 \% \\
19,2 \%\end{array}$ & $\begin{array}{c}0 \\
28,5 \% \\
35,7 \%\end{array}$ & $\begin{array}{l}<0,001 \\
\text { NS } \\
\text { NS }\end{array}$ \\
\hline $\begin{array}{l}\text { IMC }(x \pm D E) \\
\text { P Arterial Sistólica }(x \pm D E) \\
\text { P Arterial Diastólica }(x \pm D E)\end{array}$ & $\begin{array}{c}31,0(4,8) \\
132,4(18,2) \\
76,3(10,2)\end{array}$ & $\begin{array}{c}30,9(5,0) \\
132,4(18,9) \\
78,7(9,8)\end{array}$ & $\begin{array}{c}31,7(4,6) \\
132,4(17,6) \\
73,5(10,1)\end{array}$ & $\begin{array}{c}\mathrm{NS} \\
\mathrm{NS} \\
<0,05\end{array}$ \\
\hline $\begin{array}{c}\text { Analítica: Glucemia basal } \\
\text { LDL-colesterol } \\
\text { Plaquetas }\left(\times 10^{3}\right) \\
<130\left(\times 10^{3}\right)(\%) \\
\text { GOT/GPT } \geq 2 \times \text { LSN } \\
\text { Creatinina }(x \pm \mathrm{DE}) \\
\text { FGe }(x \pm \mathrm{DE}) \\
\mathrm{FGe}(<30 \mathrm{ml} / \mathrm{min}) \\
\mathrm{FGe}(30-50 \mathrm{ml} / \mathrm{min}) \\
\text { ERC (grado } 3-4) \\
\text { INR(60\% en TRT) }\end{array}$ & $\begin{array}{c}114,2(38,1) \\
109,3(27,7) \\
186,1(47,1) \\
11(11,7 \%) \\
1(1 \%) \\
1,02(0,35) \\
69,0(21,7) \\
4(4,2 \%) \\
12(12,7 \%) \\
32(34,0 \%) \\
38(40,2 \%)\end{array}$ & $\begin{array}{c}111,0(31,5) \\
109,3(28,1) \\
175,8(40,5) \\
7(13,4 \%) \\
0 \\
1,06(0,34) \\
74,2(22,4) \\
1(1,9 \%) \\
5(9,6 \%) \\
13(25,0 \%) \\
19(36,5 \%)\end{array}$ & $\begin{array}{c}118,1(45,1) \\
109,3(45,0) \\
198,9(51,9) \\
4(9,5 \%) \\
1(2,3 \%) \\
0,96(0,36) \\
62,6(19,1) \\
3(7,1 \%) \\
7(16,6 \%) \\
19(45,2 \%) \\
19(45,2 \%)\end{array}$ & $\begin{array}{l}\text { NS } \\
\text { NS } \\
<0,05 \\
\text { NS } \\
\text { NS } \\
\text { NS } \\
<0,01 \\
\text { NS } \\
\text { NS } \\
<0,05 \\
\text { NS }\end{array}$ \\
\hline
\end{tabular}

Tabla 3. Características de la muestra.

FGe: Filtrado Glomerular estimado; TRT: Tiempo en Rango Terapéutico

predictivo $^{8}$, se reclasifica a los pacientes con un punto en la escala CHADS2, de modo que si obtiene $\geq 2$ puntos en CHA2DS2-VASc existe indicación de TAO (pasando de $77,9 \%$ a $93,9 \%$ para pacientes con valores de $\geq 2$ puntos en las respectivas escalas) $)^{9}$ con el consiguiente aumento del número de pacientes en tratamiento.

Por otro lado, los pacientes con mayor riesgo de desarrollar un accidente cerebro-vascular presentan un mayor riesgo de hemorragias, que se estima con escalas tales como: HEMORR2HAGES ${ }^{10}$ y HAS-BLED ${ }^{11}$, con un máximo de 9 puntos (tabla 1), considerándose que el paciente tiene un riesgo apreciable de hemorragia cuando la puntuación es $\geq 3$ puntos.

Recientemente se han comercializado nuevos anticoagulantes que actúan sobre diferentes vías a las que lo hacen los anticoagulantes clásicos (acenocumarol o warfarina), obviando algunos de los inconvenientes de éstos y mejorando ciertos aspectos (no precisan controles analíticos, no interaccionan con alimentos, menores interacciones farmacológicas y menor riesgo de sangrado), pero con dudas no resueltas hasta el momento actual: no existencia de antídoto, limitaciones de uso en caso de alteraciones en la función renal. Ya está disponible en el mercado Dabigatrán, un inhibidor directo y reversible de la trombina, y próximamente lo estarán los inhibidores del factor Xa (Apixabán, Rivaroxabán), igualmente de administración oral. Además, es un fármaco sometido a recomendaciones específicas de la Agencia Española de Medicamentos y Productos Sanitarios en el empleo en pacientes con alteraciones en la función renal, sobre todo en mayores de 75 años ${ }^{12}$.

El objetivo principal del estudio es conocer qué pacientes con Fibrilación Auricular No Valvular (FANV) y TAO serían susceptibles de cambiar a la nueva terapia y, como objetivo secundario, conocer en cuántos de ellos sería coste-efectivo el cambio, teniendo en cuenta en ambos casos las indicaciones actuales.

\section{MATERIAL Y MÉTODOS}

Se trata de un estudio observacional, transversal y descriptivo en el ámbito de la AP (Zona de Salud de Manzanares II) que comprende la población de Membrilla y más del $50 \%$ de Manzanares. La población analizada han sido todos los pacientes de ambos sexos con factor de riesgo "anticoagulación" en el Programa Turriano, usado 
en Atención Primaria en Castilla-La Mancha. Se han considerado criterios de exclusión los siguientes: pacientes cuya indicación de TAO no era FANV, no pertenecer realmente al cupo (desplazado y/o adscripciones temporales), pacientes que aún figurando con factor de riesgo anticoagulación no seguían tratamiento con acenocumarol o se había indicado el cambio a dabigatrán en el momento de la recogida de datos, y pacientes que en el momento del estudio hubieran fallecido. El periodo de recogida de datos ha sido del 31 de enero al 31 de marzo de 2012.

Se han analizado todas las historias de la zona y de cada paciente se ha completado una hoja de recogida de datos, registrando datos sociodemográficos (edad, sexo), diagnóstico que indica la TAO, presencia de factores de riesgo cardiovascular y comorbilidades cardiovasculares, parámetros analíticos -considerando como válidos los de una antigüedad inferior a los 6 meses- $y$ antropométricos, porcentaje de control de INR, cálculo de la puntuación de las escalas CHADS2 y CHAD2DS2-VASc y HASBLED (tabla 1). También

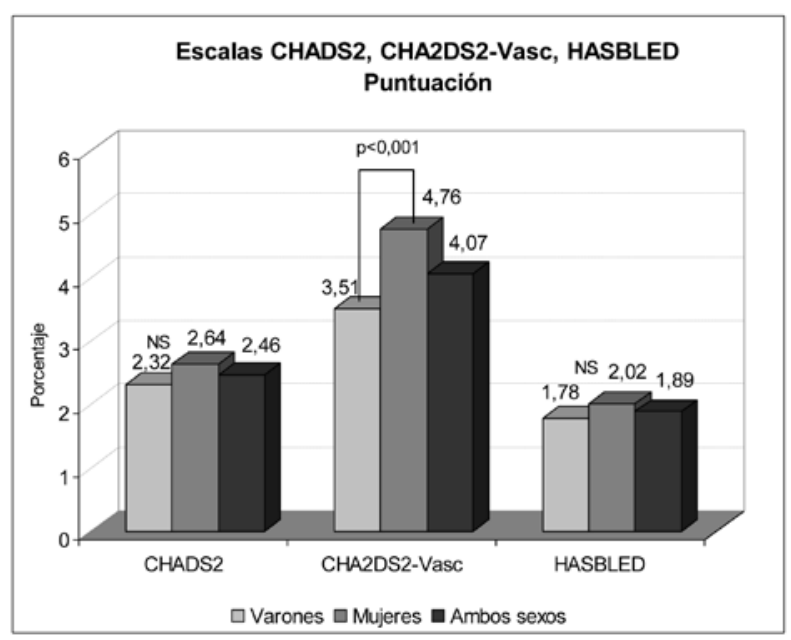

Figura 1. Puntuaciones en Escalas de Riesgo Cardioembólico (CHADS2, CHAD2DS2-Vasc) y de Sangrado (HASBLED)

se han considerado los fármacos susceptibles de interacción con dabigatrán (contraindicados: ketoconazol, itraconazol, ciclosporina, tacrolimus) o aquellos en los que hay tener cierta precaución (amiodarona, verapamilo, carbamazepina o quinidina). Con todos estos datos se ha valorado la indicación, contraindicación y/o precauciones de uso del nuevo anticoagulante: prevención del ictus y de la embolia sistémica en pacientes adultos con fibrilación auricular no valvular, con uno o más de los siguientes factores de riesgo ${ }^{13}$ :

- Ictus, accidente isquémico transitorio (AIT) o embolia sistémica (ES) previos.

- Fracción de eyección ventricular izquierda (FEVI) $<40 \%$.

- Insuficiencia cardíaca sintomática $\geq$ clase 2 en la escala NYHA

- Edad $\geq 75$ años.

- Edad $\geq 65$ años asociada a uno de los siguientes: diabetes mellitus, enfermedad coronaria 0 hipertensión.

El coste-efectividad se ha valorado según las conclusiones de Shah et $\mathrm{al}^{14}$ en el que se contempla el riesgo de ictus y de sangrado en los pacientes, de modo que: a) En pacientes de muy bajo riesgo (CHADS2 $=0$ ) la única opción coste efectiva sería la aspirina; b) Si hay un riesgo intermedio de ictus (CHADS2 = 1-2) la warfarina es la opción más coste-efectiva excepto en pacientes con muy alto de riesgo de hemorragia o muy pobre control del INR (tiempo en rango terapéutico $<57,1 \%$ ); c) En pacientes con alto riesgo (CHADS2 $\geq 3$ ) el dabigatrán a dosis de $150 \mathrm{mg} / 12$ horas es la mejor opción, salvo en pacientes con un control de INR excelente (tiempo en rango terapéutico > 72,6\%). $\mathrm{Ni}$ el dabigatrán a dosis de $110 \mathrm{mg} / 12$ horas ni la terapia dual (aspirina más clopidogrel) resultan coste-efectivos.

El tratamiento estadístico se ha realizado con el programa G-STAT 2.0, empleando la t de Student para comparación de medias y la Chi-cuadrado para comparación de porcentajes.

\section{RESULTADOS}

Con factor de riesgo "anticoagulación" había al 31 de enero de 2012 un total de 166 pacientes (93 varones y 73 mujeres), de los que se excluyeron 72 (41 varones y 31 mujeres), siendo los motivos más frecuentes: prótesis valvular (17), valvulopatía (11), trombosis venosa profunda (10), éxitus (7), miocardiopatías (6), trastornos de la coagulación (3) o encontrarse ya en tratamiento con dabigatrán (2).

De los 94 pacientes válidos con TAO por FANV -52 varones y 42 mujeres- los parámetros más relevantes son los que figuran en la tabla 3. Destaca que la mujeres son de mayor edad que los varones $(p<0,05)$ y con mayor prevalencia $(p<0,05)$ de HTA. No hay diferencias significativas en el resto de las comorbilidades (salvo EPOC), ni en IMC ni en valores de PA sistólica, pero sí en los valores de PA diastólica. En los parámetros analíticos destacan las diferencias significativas $(p<0,01)$ en el filtrado glomerular estimado ( $\mathrm{FGe}$ ), que es menor en mujeres, con un mayor porcentaje de enfermedad renal crónica (ERC) grado 3 ó 4 en mujeres que en varones $(45,2 \%$ vs $25,0 \% ; p<0,05)$. El número total de plaquetas es mayor en mujeres $(p<0,05)$. 
Hay un 40,2\% (IC95\% 30,4-51,0) de pacientes (36,5\% en varones y $45,2 \%$ en mujeres) con una media $\leq 60 \%$ del tiempo en rango terapéutico (TRT) del INR.

No se han apreciado diferencias entre sexos en la puntuación de las escalas CHADS2 ni HASBLED, pero sí en las puntuaciones de la escala CHA2DS2VASc (figura 1). No hay diferencias en el porcentaje de pacientes con $\geq 2$ puntos (escalas CHADS2 y CHA2DS2-VASc) y $\geq 3$ puntos (escala HASBLED), si bien con puntuaciones $\geq 2$ en ambas escalas de riesgo tromboembólico aumenta (del $77,9 \%$ al 93,9\%) el porcentaje de indicación de TAO (figura 2).

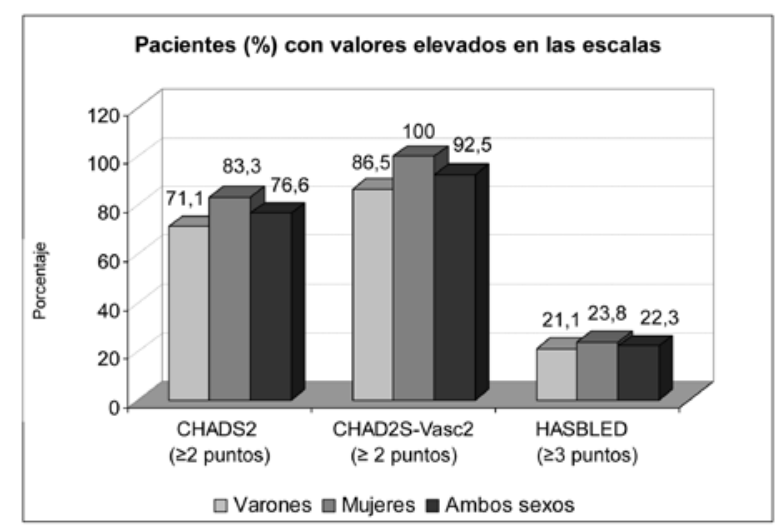

Figura 2. Pacientes con valores elevados en la escalas.

En cuanto a las indicaciones autorizadas de los nuevos anticoagulantes orales (en concreto dabigatrán), el porcentaje de pacientes a los que se le podría sustituir el acenocumarol por este nuevo tipo de fármacos es variable (figura 3 ), siendo siempre superior en mujeres que en varones (excepto en caso de ictus). Y dada la elevada prevalencia de HTA en pacientes de 65 y más años, es en este grupo de pacientes donde

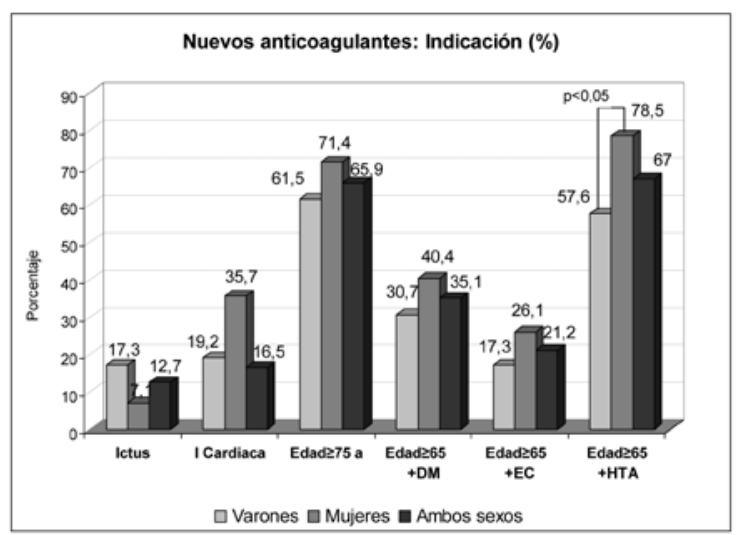

I Cardiaca: Insuficiencia Cardiaca; DM: Diabetes Mellitus; EC: Enfermedad Coronaria; HTA: Hipertensión Arterial.

Figura 3. Indicación de nuevos anticoagulantes. se obtienen los porcentajes más elevados $(67 \%$ -IC95\%: 56,5-76,3- en ambos sexos, con 57,6\% en varones y $78,5 \%$ en mujeres) siendo el único donde hay diferencias significativas entre sexos $(p<0,05)$.

Estarían contraindicados en los pacientes con $\mathrm{FGe}$ $<30 \mathrm{ml} / \mathrm{min}, 1,9 \%$ en varones y $7,1 \%$ en mujeres, pero sin significación (figura 4) y habría que tener cierta precaución en su uso en un porcentaje variable, siendo más frecuente las alteraciones de la función renal $(16,6 \%)$ en mujeres, la trombopenia $(13,4 \%)$ en varones, y en un $5 \%$ en caso de indicación previa de amiodarona o verapamilo, aunque sin diferencias entre sexos en todos los casos (figura 4).

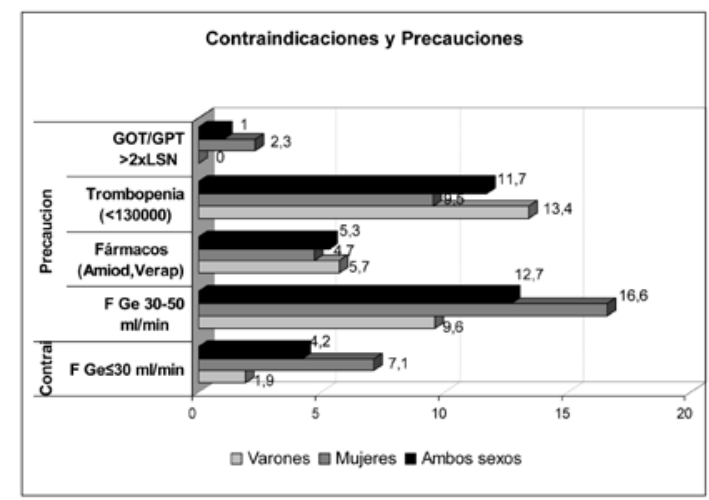

GOT/GPT: Transaminasa Glutámico Oxalacética/Transaminasa Glutámico Pirúvica; LSN: Limite superior de la normalidad; Amiod: Amiodarona; Verap: Verapamil; FGe: Filtrado Glomerular estimado (MDRD)

Figura 4. Contraindicaciones y Precauciones.

En cuanto al coste-efectividad que supondría el cambio, éste podría ser coste efectivo hasta en un $32,9 \%$ (IC95\%: 23,6-43,4) del total de los pacientes $(28,7 \%$ en varones y $38,5 \%$ de las mujeres) (figura 5) ya que a igualdad de TRT se dobla cuando el

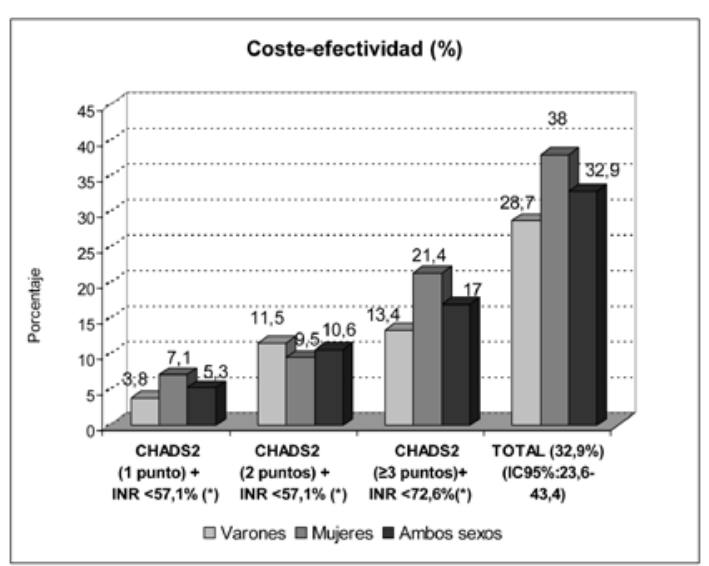

(*) Porcentaje de Tiempo en Rango Terapéutico del INR.

Figura 5. Coste-efectividad (según escala CHADS2 y porcentaje de tiempo en rango terapéutico del INR). 
CHADS2 es de dos puntos frente a uno, siendo mayor si CHADS2 es $\geq 3$ puntos y el INR es $\leq 72,6 \%$.

\section{DISCUSIÓN}

Comparando este estudio con otros, la edad media de los pacientes es superior a los incluidos en el estudio RE-LY ${ }^{15}$, donde había un $64,3 \%$ de varones frente a un $55,3 \%$ en este estudio que nos ocupa. La prevalencia de factores de riesgo, sobre todo hipertensión, es elevada, aunque inferior a la referida en otros estudios ${ }^{3,16}$, siendo en las mujeres donde los valores son mayores (excepto tabaquismo y EPOC que predomina en varones). Otras enfermedades están presentes en unos porcentajes similares a estudios como el ValFAAP $^{3}$, excepto diabetes, superior a los datos del estudio RE-LY.

En los parámetros analíticos destaca la mayor prevalencia de ERC en mujeres, con un menor FGe, lo que sin duda puede condicionar la indicación/ contraindicación de los nuevos anticoagulantes, al igual que la presencia de trombopenia (más frecuente en varones)

Los valores medios de la escala CHADS2 de los pacientes ( $y$ el porcentaje con puntuación $\geq 2$ puntos) objeto de estudio han sido superiores a los del estudio RE-LY' ${ }^{15}$. Hay un $22,3 \%$ con riesgo de sangrado según la escala HASBLED, y un $40,2 \%$ no tienen el INR dentro del tiempo del rango terapéutico, superior al de los participantes españoles (34\%) del estudio RE-LY. Estas diferencias podrían explicarse por la mayor edad de los pacientes que influye en las puntuaciones de las escalas y a la mayor prevalencia de diabetes mellitus. Hay diferencias en la escala CHA2DS2VASc entre sexos (mayor en mujeres), pero considerando que uno de los puntos corresponde por ser de sexo femenino, podría explicar la diferencia en las puntuaciones.

Según las indicaciones actualmente aprobadas podría estar indicado hasta en dos de cada 3 pacientes, por ser de $\geq 65$ años con $H T A o \geq 75$ años.

Estaría por otra parte contraindicado en caso de $\mathrm{FGe}<30 \mathrm{ml} / \mathrm{min}$ (4,2\% de los pacientes), y debería indicarse con precaución en un porcentaje variable según la condición, siendo nuevamente el valor del FGe el que en mayor porcentaje aconseja cierta precaución, seguida por la trombopenia y el uso concomitante de fármacos con acción sobre la glucoproteína $P$, aunque sin significación entre sexos. La alteración en la función renal ha de ser tenido siempre en cuenta ya que, a excepción de antecedentes de ictus, es en las mujeres donde la indicación presenta los porcentajes mayores, y al mismo tiempo las mujeres tienen más alteraciones en la función renal, siendo aconsejable la individualización de la indicación del cambio de anticoagulante.

La aplicación de los criterios del estudio $^{14}$ a los pacientes de este estudio indican que sería coste-efectivo en un porcentaje variable (según puntuación CHADS2 y porcentaje de TRT del INR), hasta un total del $32,9 \%$. Hemos de tener presente el impacto económico, ya que considerando una dosis media de $4 \mathrm{mg} /$ día de acenocumarol junto a 13 determinaciones anuales de INR, el coste global (sin incluir la amortización de la máquina) es de $273,48 €$ frente a 1197,2 € del coste de dabigatrán ${ }^{17}$, por lo que el cambio a todos los pacientes en los que pueda estar indicado incrementaría notablemente el gasto, siendo por tanto coste efectivo sólo cuando concurren otras circunstancias como las anteriormente referidas. Sería aconsejable mantener el tratamiento con acenocumarol como mejor opción terapéutica cuando el paciente tenga un buen control del INR.

Se puede concluir que el nuevo anticoagulante oral podría estar indicado hasta en $2 / 3$ de los pacientes con FANV, sobre todo en aquellos mayores de 65 años con HTA o en los mayores de 75 años. Estarían contraindicados en aproximadamente un $4 \%$ de los casos, y habría que usarlos con precaución en uno de cada ocho pacientes, por alteración en la función renal o trombopenia, y en un $5 \%$ por uso concomitante con otros fármacos (sobre todo amiodarona o verapamilo). Su empleo podría ser coste-efectivo hasta en uno de cada 3 pacientes, estando en relación directa con la puntuación de la Escala CHADS2 y el TRT del INR.

Hay que considerar una limitación al trabajo: se han analizado los pacientes con factor de riesgo "anticoagulación" y fibrilación auricular no valvular, por lo que pueden no haberse incluido pacientes con FA a los que la aplicación de las escalas resultase la indicación de TAO: no olvidemos la conclusión del estudio Val-FAAP ${ }^{3}$ de que el "tratamiento anticoagulante en la fibrilación auricular atendida en Atención Primaria dista mucho de ser óptimo". Dadas las características evolutivas de la FANV habría que considerar la re-evaluación del riesgo de complicaciones tromboembólicas y de sangrado en cada uno de los pacientes con diagnóstico de FA con la finalidad de adecuar el tratamiento (antiagregante o anticoagulante) según las escalas de riesgo tromboembólico y de sangrado. 


\section{BIBLIOGRAFÍA}

1. Navarro JL, Cesar JM, Fernández MA, Fontcuberta J, Reverter JC, Gol-Freixa J. Morbilidad y mortalidad en pacientes con tratamiento anticoagulante oral. Rev Esp Cardiol. 2007; 60(12):1226-32.

2. Oral anticoagulant control [editorial]. The Lancet. 1987; 488-9

3. Barrios V, Calderón A, Escobar C, De la Figuera M. Pacientes con fibrilación auricular asistidos en consultas de atención primaria. Estudio Val-FAAP. Rev Esp Cardiol. 2012; 65 (1): 47-53.

4. Kato T, Yamashita T, Sagara K, linuma H, Fu LT. Progressive nature of paroxysmal atrial fibrillation. Observations from a 14-year follow-up study. Circ J. 2004; 68:568-72

5. Camm AJ, Kirchhof P, Lip GY, Schotten U, Savelieva I, Ernst $S$, et al. Guías de práctica clínica para el manejo de la fibrilación auricular ( $2^{\mathrm{a}}$ edición corregida). Rev Esp Cardiol. 2010; 63: 1483. e1-83.

6. Ogilvie IM, Newton N, Welter SA, Cowell W, Lip GY. Underuse of oral anticoagulants in atrial fibrillation: a systematic review. Am J Med. 2010; 123: 638-45.

7. Gage BF, Waterman AD, Shannon W, Boechler M, Rich MW, Radford MJ. Validation of clinical classification schemes for predicting stroke: results from the National Registry of Atrial Fibrillation. JAMA. 2001; 285: 2864-70.

8. Banerjee A, Marín F, Lip GY. Avances incompletos en la estratificación del riesgo de ictus en la fibrilación auricular. Rev Esp Cardiol. 2011; 64 (8):639-641.

9. Rodríguez-Mañero $\mathrm{M}$, Cordero $\mathrm{A}$, Bertomeu-González V, Moreno-Arribas J, Bertomeu-Martínez V, Mazón P, et al. Impacto de los nuevos criterios para el tratamiento anticoagulante de la fibrilación auricular. Rev Esp Cardiol. 2011; 64(8):649-653.

10. Gage B, Yan Y, Milligan PE, Waterman AD, Culverhouse R, Rich MW, Radford MJ. Clinical Classification schemes for predicting hemorrhage: Results from the National Registry of Atrial Fibrillation (NRAF) Am Heart Journal. 2006; 151(3): 713-719
11. Pisters R, Lane DA, Nieuwlaat R, de Vos CB, Crijns HJ, Lip GY. A novel user-friendly score (HAS-BLED) to assess 1-year risk of major bleeding in patients with atrial fibrillation: the Euro Heart Survey. Chest. 2010: 138: 1093-100.

12. Dabigatrán (Pradaxa $\AA$ ) y Riesgo de hemorragia: nuevas recomendaciones de vigilancia de la función renal. Disponible en http://www.aemps.es Fecha de publicación: 27 de Octubre de 2011.

13. PRADAXA®: Ficha técnica del producto. Disponible en http://www.ema.europa.eu.

14. Shah SV, Gage BF. Cost-effectiveness of Dabigatran for Stroke Prophylasis in Atrial Fibrilattion. Circulation. 2011;123:2562-2570.

15. Connolly SJ, Ezekowitz MD, Yusuf S, Eikelboom J, Oldgren $\mathrm{J}$, Parekh A et al. Dabigatran versus warfarin in patients with atrial fibrillation (the RE-LY trial) N Engl J Med. 2009; 361:1139-51.

16. Mazon P. Cordero A, González-Juanatey JR, Galve E, Lekouna I, Facila L et al. Investigadores del grupo CARDIOTENS. Impacto de la hipertensión arterial en la cardiopatía en España: evolución de la última década. Rev Esp Cardiol. 2010; 63 Suppl 3:53 (A427)

17. Galindo Rueda, M. Dabigatran. Hoja de evaluación de medicamentos. Servicio Murciano de Salud. Volumen XII. Número 2. Octubre 2011. 